\title{
To design versus to understand design: the role of graphic representations and verbal expressions
}

Zuhal Ulusoy, Bilkent University, Department of Landscape Architecture and Urban Design, Ankara, 06533 Turkey

While the primary objective of design education is essentially teaching how to design, the process of understanding a design product is another important and obvious goal of design education. Here, the aim is to find out how the capacity of the students to design relates to their capacity to understand and evaluate design. In a survey done in the freshman studio, students were asked to diagrammatically express the design ideas of the projects they were shown, to criticize the projects verbally and to grade them. Later, the diagrams produced by the students, their verbal evaluations of the projects, the grades they have assigned to these projects, and their own projects' jury grades are compared to find out the interaction among these variables. (C) 1999 Elsevier Science Ltd. All rights reserved

Keywords: design education, evaluation, graphic representation, conceptual design

$\mathrm{T}$ This study is based on the assumption that 'to design' and 'to understand design' are two related but distinct processes. The activity of design necessitates making decisions, comparing alternative routes to take to decide on one, and thinking in abstract terms for conceptualizing; thus, it is synthetic. On the other hand, the activity of understanding design is similar to reading, necessitates deciphering the thought process of the designer, making abstractions of relations, relating the design product to other examples; hence, it is analytical. As such, the common denominator between the two processes is the capacity of abstraction, ability of thinking in abstract terms. Yet, the nature and the medium of abstraction may not be the same for students with different tendencies. Here, it is argued that while the act of designing (synthesis) is primarily related with the ability of making visual abstractions (graphic analysis), 'correctly' judging a design product is related to the ability of making 
1 Goldschmidt, G 'The dialectics of sketching' Creativity Research Journal Vol 4 No 2 (1991) pp 123-143

2 Schön, D 'The architectural studio as an exemplar of education for reflection-in-action' Journal of Architectural Education Vol 38 No 1 (1984) pp 2-9 3 Schön, D The reflective practitioner Avebury, Aldershot (1991)

4 Goldschmidt, G 'On visual design thinking: the vis kids of architecture' Design Studies Vol 15 No 2 (1994) pp 158-174 articulated verbal evaluations (verbal analysis). The reasoning behind this argument is that judgements involve verbal associations such as good, bad, successful.

In the following survey, making graphical analysis is considered to reflect a student's ability in visual thinking, whereas verbally analyzing design products is taken as representing his/her ability in linguistic thinking. It is suggested that the relation between to design and to understand design needs to be discussed in terms of the distinction and interaction between these two faculties.

\section{Objective of design education}

Design education essentially deals with teaching how to design, moreover, rather ideally, with guiding students to discover their own ways of designing. Students enrol in design schools mostly without being evaluated on the basis of their ability to design. Yet, they are expected to show a reasonable level of performance in order to graduate. Much of secondary education they receive does not promote visual thinking, whereas design education, by its nature, requires the students to be able to think and conceptualize visually.

While designing, designers utilize various techniques, consciously or intuitively, like sketching both to record and to generate ideas ${ }^{1}$. The process of designing involves sequential, and in some cases simultaneous, stages of making decisions and criticism, a process Schön refers to as 'reflectionin-action ${ }^{2,3}$. These indicate the incorporation of interactive imagery and visual thinking to arrive at creative solutions which are argued to be 'perfectly rational and highly systematic' (Goldschmidt ${ }^{4}, p$ 166) ways of reasoning.

Parallel to the objective of teaching design is a second, almost as important and obvious objective: teaching the process of understanding and criticizing a design product. In the case of architectural design, this may mean visually decoding the relations among spaces, and/or verbally criticizing their performance with respect to a set of criteria. Within the context of understanding design, graphic expressions of a design product done by someone other than the designer himself/herself can be taken as cases of visual thinking or visual conceptualization, as they involve visual interpretation. These visual interpretations may be highly abstract and conceptual in nature, such as deciphering the meanings associated with spaces and expressing the 'figural concept' of design (Goldschmidt ${ }^{4}, \mathrm{p}$ 174). They may as well be quite descriptive, representing the components of spaces in the form of perspective drawings. Similarly, verbal criticism of design products 
5 Suwa, $M$ and Tversky, B 'What do architects and students perceive in their sketches? A protocol analysis' Design Studies Vol 18 No 4 (1997) pp 385403

6 Akin, Ö 'Representation and architecture'. In Akin, Ö and Weinel E F (eds) Representation and architecture Information Dynamics Inc., Silversprings MD (1982) pp 1-26

7 Jackendoff, $\mathbf{R}$ 'On beyond zebra: the relation of linguistic and visual information' Cognition Vol 26 No 2 (1987) pp 89-114 involve interpretations that reflect linguistic thinking or conceptual reasoning. In this context, it is argued that the linguistic faculties of students are influential on the process of understanding, criticizing and evaluating design, that is, on their critical judgements of projects.

\section{Graphic representations and verbal expressions as analytical tools}

Graphic representations have a unique significance in design. Since design primarily deals with the creation of forms, it heavily relies on visual imagery and those tools that help to express $i^{4}$. Sketches are useful not only as memory aids to record ideas to be recollected but also as inseparable tools of design process for crystallizing design ideas and for generating further thoughts 5 .

In addition to their function in the design process, sketches and other visual tools are helpful for analyzing the designs made by others. Diagrams as abstract expressions of the design idea are such examples. Locational relations among spaces, physical connections and adjacencies, as well as more abstract and non-visual aspects, can be traced and represented graphically. This valuable use of sketches in analyzing a design product and deciphering its design idea can be viewed as another form of 'reflectionin-action', action being the act of drawing to understand the inherent nature of a project. What the observer does in such a case is selectively drawing the formal and conceptual characteristics of a project, thus graphically representing his/her interpretation of it $^{6}$.

Another category of analytical tools used in criticism and evaluation of design is that of verbal expressions, representing linguistic thinking. Again the critique selects formal and conceptual aspects that characterize a design product, discovers meanings associated with it and interprets them verbally, thus translates the formal relations that he/she reads into linguistic expressions ${ }^{7}$.

\section{Case study}

The conducted survey is based on the idea that visual and linguistic abilities of students can be traced in their performance in the design studio. The hypothesis is that there is a direct relation between the students' ability to design (synthesis) and their ability to make graphic analyses, and also, between the students' evaluations of others' projects and their ability for verbal analyses. Here, making graphic analysis is considered to reflect a student's ability in visual thinking, whereas verbally analyzing design products is considered to represent his/her ability in linguistic thinking. Thus, the argument is that while design is related mostly with visual thinking, 
understanding design is related with linguistic faculties. In order to test this, a research is conducted during the final juries in the Department of Landscape Architecture and Urban Design at Bilkent University, Turkey.

\subsection{Data categories}

The group of students who took part in the study were all freshman students at the end of their first semester in design studio. A total of 32 students who had submitted projects for the jury were asked to participate in the research during the jury. They were given an evaluation form on which the names of the students and the criteria for evaluation were listed. For each project, the students were asked to express its design idea diagrammatically, to write down their criticisms of the project briefly and to give a letter grade (from $\mathrm{A}$ to $\mathrm{F}$ ).

The data consisted of four categories:

(1) Graphic representations (diagrams) of the design idea for individual projects drawn by each student, as a measure of his/her ability for graphic analysis;

(2) Written criticisms for each project, to measure each student's ability for verbal analysis;

(3) Correlation between the grades given by each student and the jury, to understand his/her ability to evaluate projects 'correctly', within the given set of criteria;

(4) Grades each student received for his/her project, representing the student's ability to design.

\subsection{Evaluation of data}

The graphic representation of a project is taken simply as a diagram of the relation among spaces. The diagrams produced by the students were evaluated on the basis of their representativeness of the design idea, using a three-point scale. The total points each student received was divided by the number of projects he/she had evaluated. This way, it was possible to obtain a score between 0.00 and 1.00 for each student which is accepted to measure his/her ability to make graphic analysis. The diagrams were rather naive, mostly because the students were at the end of their first semester and not very familiar with sketching as a tool. Since the idea is the representativeness of these diagrams, the quality of the drawings were not particularly considered.

The verbal criticisms of the projects done by the students are evaluated on the basis of the articulation of verbal expression and the 'truthfulness' of evaluation, again using a three-point scale. Similar to graphic 
representations, the total points each student received was divided by the number of projects he/she had evaluated, which became the score of his/her ability of making verbal analysis.

It is assumed that jury grades reflect an 'objective' evaluation of the projects with respect to the given design criteria. Thus, the closer the grade assigned by a student gets to the jury grade, the better is his/her judgement of the project. Correlation between the grades given by a student for each project and the grades the jury gave to the same projects was done to see the degree of match between the judgement of the jury and that of the student. The scores students got as the result of this correlation test ranged between 0.58 and 0.99 , reflecting direct relation between their assessments of the projects and the jury grades at 0.025 level of significance.

Finally, the grade each student received from the jury for his/her project is taken as a measure of his/her ability in design, in other words, his/her ability to synthesize various aspects of a design problem. The design grades of the students who participated in the study ranged between $0.0(\mathrm{~F})$ and $3.3\left(\mathrm{~B}^{+}\right)$.

Four data categories (Table 1) were studied to test the previously stated hypothesis that, while design is related mostly with visual thinking, understanding design is related with linguistic faculties. The scores of each student in pairs of data categories were plotted as scatter graphs. A total of six such graphs showing all the possible combinations were produced and studied. Performing simple curve fit (first order), it is seen that relation between 'graphic representation' and 'grade received from the jury' has

Table 1 Categories of data

\begin{tabular}{|c|c|c|c|c|}
\hline & Verbal expression & $\begin{array}{c}\text { Graphic } \\
\text { representation }\end{array}$ & $\begin{array}{l}\text { Correlation with } \\
\text { jury grade }\end{array}$ & Jury grade received \\
\hline Başak & 0.944 & 0.444 & 0.945 & 1.3 \\
\hline Tülin & 0.432 & 0.636 & 0.863 & 3 \\
\hline Uğur & 0.222 & 0.556 & 0.934 & 3.3 \\
\hline Övünç & 0.539 & 0.654 & 0.581 & 2 \\
\hline Hakan & 0.438 & 0.406 & 0.775 & 1.3 \\
\hline Ulaş & 0.125 & 0.313 & 0.809 & 0 \\
\hline Ayşe & 0.273 & 0.318 & 0.874 & 1.7 \\
\hline Ardan & 0.778 & 0.556 & 0.757 & 1.3 \\
\hline Eda & 0.375 & 0.083 & 0.642 & 1.7 \\
\hline Hande & 1 & 0.3 & 0.952 & 1 \\
\hline Erkan & 0.211 & 0.263 & 0.799 & 1.7 \\
\hline Alper & 0.346 & 0.308 & 0.749 & 2.7 \\
\hline
\end{tabular}


the largest value, as shown in Table 2. Second in this ranking is the relation between 'verbal expression' and 'correlation with jury grade'. Thus, although none of the combinations reflect statistically significant correlations between pairs of categories, their relative position suggests a higher relation between the categories that represent visual thinking and design ability, and between linguistic thinking and 'correct' judgement of projects.

Next, polynomial curve fit (second order) was applied to the scatter graphs. Ranking the results, it was found that two pairs of categories, that is, 'correlation with the jury grade' with 'verbal expression', and, 'correlation with the jury grade' with 'graphic representation' had the highest values among the six (Table 3). This suggests, in addition to the expected outcome that understanding design is related with verbal thinking, its relation with visual thinking is also observable. It must be noted that, although these relations are not statistically significant, they represent correlations that are relatively higher compared to those of other combinations.

\section{Discussion and conclusion}

The starting point of this study has been that students with verbal abilities and those with graphic abilities tend to reflect this difference onto various aspects of their studio performance. Thus, not only their design ability, hence synthetic thinking, varies but also their analytical thinking, in verbal and graphical terms, differs. It is generally expected that those who can express their design ideas using visual tools can be better designers. What

Table 2 Correlations between categories

\begin{tabular}{lcccc}
\hline & $\begin{array}{c}\text { Verbal } \\
\text { expression }\end{array}$ & $\begin{array}{c}\text { Graphic } \\
\text { representation }\end{array}$ & $\begin{array}{c}\text { Correlation with } \\
\text { jury grade }\end{array}$ & $\begin{array}{c}\text { Jury grade } \\
\text { received }\end{array}$ \\
\hline Verbal expression & 1.000 & 0.200 & 0.243 & -0.219 \\
Graphic representation & & 1.000 & 0.056 & 0.392 \\
Correlation w/jury grade & & 1.000 & 0.020 \\
Jury grade received & & & 1.000 \\
\hline
\end{tabular}

Table 3 Results of second order correlation between categories

'Verbal expression' and 'Correlation with jury grade'

'Graphic representation' and 'Correlation with jury grade'

'Verbal expression' and 'Jury grade received'

'Graphic representation' and 'Jury grade received'

'Verbal expression' and 'Graphic representation'

'Correlation with jury grade' and 'Jury grade received'

$$
\begin{aligned}
& R=0.68 \\
& R=0.62 \\
& R=0.52 \\
& R=0.50 \\
& R=0.41 \\
& R=0.13
\end{aligned}
$$


would be interesting would be to find out that these better designers are also good at visual analysis, that is, extracting formal characteristics of the projects they study. Keeping in mind that the results of the statistical analysis of the above survey should be approached with reservation, it can still be stated that the relation of graphic analysis with design ability, and the relation of ability to judge projects 'correctly' with both verbal and graphic analyses are higher compared to the relations between other categories of data. This translates that, as opposed to design's relation mostly with visual thinking, understanding design incorporates both visual and linguistic faculties.

It is possible to interpret these results in terms of the processes of translation that occur between visual and linguistic faculties. Verbal analysis of a project involves a translation from visual/formal medium to the medium of language. Since the students who took part in this survey are at the beginning of their design education, they are more familiar with expressing their ideas verbally than visually. The process in visual analysis, on the other hand, does not involve a translation but requires an abstraction; a process that occurs within the same visual/formal medium. Designing, thus synthesis, however, requires the end-product to be represented in visual/formal medium, after a process of continuous translations between linguistic and visual faculties. These translations in design activity support the view that 'spatial thinking depends on aspects of both' of these faculties (Jackendoff ${ }^{7}, \mathrm{p}$ 110). Compared to verbal and visual analyses, design is a more complex process, both due to the incorporation of numerous translations, and due to the difficulties of expressing ideas visually. Thus, not finding a relation between verbal analysis and design does not necessarily mean that design is not related with linguistic thinking, but that those who are better designers can express themselves better visually than verbally. Judgements, on the other hand, are more straightforward as long as the observer can analyze a project, either verbally or visually, on the basis of its performance in resolving the design problem.

Most of the literature on design cognition focuses on rationalizing and deciphering the cognitive processes involved in design activity, investigating the role of verbal and graphic thinking in the process. The interaction between verbal and visual conceptualization is far more complex than can be discussed within the limited scope of this study. Yet, the significance of this interaction also in analyzing design products should not be overlooked. Here an attempt has been made to incorporate design (synthesis) and understanding design (analysis) with faculties of verbal and visual thinking, within the context of a case study. 
8 Eisentraut R and Günther J 'Individual styles of problem solving and their relation to representations in the design process'. In Akin Ö and Sağlamer G (eds) First International Symposium on Descriptive Models of Design Proceedings ITU, Istanbul, Turkey (1996) pp 53-71
Suggestions can be made for improving the research in order to arrive at stronger supports for the hypothesis and further results. Firstly, the sample size should be increased. In the survey, the number of usable evaluation forms was smaller (12 forms) than what was initially intended. Not all the students filled out the forms, and not for all the projects. The smallness of sample size has limited the chance to conduct a more rigorous statistical analysis, reducing the reliability of results. Secondly, it will be more sound if the measurement of a student's design ability depends on his/her performance in more than one project. And thirdly, conducting a similar survey with students from higher level studios might enable a comparison to discuss the effects of design education, first on their ability to design and to understand design, and, second on their use of visual and graphic faculties for analysis and synthesis. Such a comparison can lead to searching for innovative approaches to design education which recognizes the different tendencies and abilities among students, supporting their individual styles of problem solving ${ }^{8}$, and help to develop methods of improving their abilities. 